\title{
A Professional Development Model for Medical Laboratory Scientists Working in the Core Laboratory
}

\author{
FAHEEM A. ALI, LILA A. PULIDO, MELINDA N. GARZA, MEGAN H. AMERSON, BRANDY \\ GREENHILL, KRYSTYNA N. BROWN, SHARI K. LIM, VENKATESARA R. MANYAM, HANNAH \\ N. NGUYEN, CARRIE C. PRUDHOMME, LAURA E. REGAN, WILLIE R. SIMS, AFAMEFUNA U. \\ UMEH, ROSEMARY WILLIAMS, PATRICIA K. TILLMAN, PETER C. HU
}

\begin{abstract}
The Division of Pathology and Laboratory Medicine at The University of Texas MD Anderson Cancer Center has implemented a professional development model designed to further the education, expertise, and experiences of medical laboratory scientists in the core laboratory. The professional development model (PDM) has four competency levels: Discovery, Application, Maturation and Expert. All levels require the medical laboratory scientist to learn new skill sets, complete task and projects, and meet continuing education and certification requirements. Each level encourages personal development, recognizes increased competencies, and sets high standards for all services provided. Upon completion of a level within a given timeframe, the medical laboratory scientist receives a salary adjustment based on the competency level completed.
\end{abstract}

ABBREVIATIONS: PDM - professional development model; CAP - College of American Pathologists; LIS laboratory information system; ESR - erythrocyte sedimentation rate; CE - continuing education; CSF cerebrospinal fluid; hCG - human chorionic gonadotrophin; $\mathrm{H} \& \mathrm{H}$ - hemoglobin and hematocrit; QA - quality assurance; PI - performance improvement; and QC - quality control.

INDEX TERMS: Professional development model, hematology, chemistry, laboratory

Clin Lab Sci 2012;25(2):67

Faheem A. Ali, School of Health Professions, The University of Texas, MD Anderson Cancer Center, Houston, TX 77030
Lila A. Pulido, Division of Pathology and Laboratory Medicine, The University of Texas, MD Anderson Cancer Center, Houston, TX 77030

Melinda N. Garza, School of Health Professions, The University of Texas, MD Anderson Cancer Center, Houston, TX 77030

Megan H. Amerson, School of Health Professions, The University of Texas, MD Anderson Cancer Center, Houston, TX 77030

Brandy Greenhill, School of Health Professions, The University of Texas, MD Anderson Cancer Center, Houston, TX 77030

Krystyna N. Brown, Division of Pathology and Laboratory Medicine, The University of Texas, MD Anderson Cancer Center, Houston, TX 77030

Shari K. Lim, Division of Pathology and Laboratory Medicine, The University of Texas, MD Anderson Cancer Center, Houston, TX 77030

Venkatesara R. Manyam, Division of Pathology and Laboratory Medicine, The University of Texas, $M D$ Anderson Cancer Center, Houston, TX 77030

Hannab N. Nguyen, Division of Pathology and Laboratory Medicine, The University of Texas, $M D$ Anderson Cancer Center, Houston, TX 77030

Carrie C. Prudhomme, Division of Pathology and Laboratory Medicine, The University of Texas, $M D$ Anderson Cancer Center, Houston, TX 77030 


\section{CLINICAL PRACTICE}

Laura E. Regan, Division of Pathology and Laboratory Medicine, The University of Texas, MD Anderson Cancer Center, Houston, TX 77030

Willie R. Sims, Division of Pathology and Laboratory Medicine, The University of Texas, MD Anderson Cancer Center, Houston, TX 77030

Afamefuna $U$. Umeh, Division of Pathology and Laboratory Medicine, The University of Texas, $M D$ Anderson Cancer Center, Houston, TX 77030

Rosemary Williams, Division of Pathology and Laboratory Medicine, The University of Texas, $M D$ Anderson Cancer Center, Houston, TX 77030

Patricia K. Tillman, Division of Pathology and Laboratory Medicine, The University of Texas, MD Anderson Cancer Center, Houston, TX 77030

Peter C. $\mathbf{H u}$, School of Health Professions, The University of Texas, MD Anderson Cancer Center, Houston, TX 77030

Address for Correspondence: Peter Hu, PhD, 1515 Holcombe Blvd, Unit 2, Houston, TX 77030, 713-5633095,pchu@mdanderson.org

\section{INTRODUCTION}

The University of Texas MD Anderson Cancer Center is dedicated to providing excellence in the care of cancer patients from around the world. By placing an emphasis on setting higher standards in the clinical laboratory, MD Anderson is working to continuously improve the overall prevention, diagnosis, and treatment of cancer and cancer-related diseases. To achieve this goal, the Department of Pathology and Laboratory Medicine has created a framework for developing professionals in the laboratory by helping to further advance the education, expertise, and experience of its laboratory personnel. The Core Lab PDM was developed in 2007, when the lab was located in a different area, and not automated. In 2009 the Core Lab moved into space that had been renovated to accommodate an automation line. This changed the workflow, and initiated the revision of the PDM. The Core Lab PDM committee used the current PDM as the starting point, and incorporated aspects of the Cytogenetics Lab PDM and the Molecular Lab PDM that were applicable. ${ }^{1,2}$ Building on the previously adopted professional development model (PDM) for cytogenetic technologists, the core laboratory at MD Anderson has implemented a professional development model to advance the quality of service for chemistry, hematology, coagulation, and urinalysis testing. The professional development model is designed to increase the skill sets, proficiency, and professionalism of the medical laboratory scientists by allowing them to experience and understand all aspects of the laboratory from the basics task through management skills.

The PDM is designed with four competency levels: Discovery, Application, Maturation, and Expert. A person's competency is defined as their ability to integrate their knowledge, attitudes, skills, behaviors, and standards of practice into their actual performance. ${ }^{1,2}$ Each competency level of the PDM requires medical laboratory scientist to learn a new skill set, become proficient in each skill set, and master the skill set through experience. Medical laboratory scientists are trained in the fundamental skills (Discovery level) through the upper-level managerial skills (Expert level). Each level is designed to enable the medical laboratory scientist to build on the knowledge they acquired from the previous levels. Medical laboratory scientists are continuously building upon their previous expertise, from operating the automation of the laboratory and performing quality control to managing inventory and preparing for the College of American Pathologists (CAP) inspections. In addition, completion of each level requires continuing education and perhaps certification. Finally, the PDM is designed to allow medical laboratory scientists an opportunity to progress in the laboratory and to allow laboratory supervisors to recognize employees for their accomplishments.

\section{Progression Through the Competency Levels}

To progress through each level, medical laboratory scientists must learn new skill sets, complete certain tasks or projects fulfill continuing education requirements, and meet certification requirements. Medical laboratory scientists are allocated a minimum and maximum time frame to learn, perform, practice, troubleshoot, and become proficient in each skill set. The medical laboratory scientist's mastery of the skill set is validated through testing by their laboratory supervisor. Table 1 lists the minimum and maximum time frames for completing each of the four levels. 


\section{CLINICAL PRACTICE}

Table 1. Time to Complete Each Competency Level

\begin{tabular}{ccc}
\hline Competency Level & $\begin{array}{c}\text { Minimum Time } \\
\text { (Months) }\end{array}$ & $\begin{array}{c}\text { Maximum Time } \\
\text { (Months) }\end{array}$ \\
\hline Discovery & 12 & 18 \\
Application & 18 & 24 \\
Maturation & 18 & 24 \\
Expert & N/A & N/A \\
\hline
\end{tabular}

The competency levels have minimum time limits to ensure that the medical laboratory scientist does not progress too quickly through a level before fully mastering its objectives. Similarly, a maximum time limit is given to prevent the medial laboratory scientist from becoming stagnant at a certain level. All medical laboratory scientists are required to complete each competency level within the minimum and maximum time limits. Medical laboratory scientists who do not comply with these requirements may be disciplined, up to and including termination. A time frame is not given for the Expert level because this level is optional but encouraged. The title of "Senior Medical Technologist" is given when medical laboratory scientists complete the Maturation level.

In addition, medical laboratory scientists who have been with MD Anderson for more than 20 years have been allowed the option to be grandfathered from meeting the requirements of the PDM. However, medical laboratory scientists who have chosen to be grandfathered are ineligible to receive the additional salary-related adjustment specified in the PDM.

\section{Procedure for Implementing the PDM}

In the Discovery level (Table 2), medical laboratory scientists are required to become proficient in the use of automated equipment in the Core Laboratory, including performing patient-sample testing, quality control, maintenance, and troubleshooting. Additionally, the medical laboratory scientist must learn to operate the laboratory information system (LIS) and interpret patient data.

After mastering these core principles, the medical laboratory scientists proceed to the Application level (Table 3) where they build on their knowledge gained in the Discovery level. In the Application level, medical laboratory scientists continue to learn how to use automated equipment in the Core Laboratory and further advance their understanding by learning the methodologies used when running each instrument. In addition, medical laboratory scientists rotate through manual testing stations performing cell differential, reticulocyte counts, and determining erythrocyte sedimentation rates (ESR). Furthermore, medical laboratory scientists are trained in supervisory skills, including managing inventory, assisting with CAP proficiency testing, and completing an assigned project.

In the Maturation level (Table 4), medical laboratory scientists continue to gain supervisory experience, including assisting with the CAP inspection, performing semiannual linearity and correlation checks and revising or writing new procedures. In addition, medical laboratory scientists are also required to complete two of seven optional projects with approval from the laboratory manager.

Lastly, the Expert level (Table 5) is optional but encouraged, as it prepares medical laboratory scientists for possible managerial positions. The Expert level involves mastering and incorporating all previous knowledge and applying it from a managerial perspective. Requirements for the Expert level include attending management classes, working closely with managerial staff, and assuming a managerial role when necessary. Upon completing Expert level, medical laboratory scientists should understand the responsibilities of a manager and understand the skills needed to successfully run a laboratory.

Biannually, a laboratory supervisor meets individually with his or her staff to assess their current progress in the PDM and to evaluate the objectives they have completed. Evaluations are in the form of either a written exams or performance of a specific task. The supervisor assesses whether the medical laboratory scientist has completed all the objectives for his or her current level and is ready to progress or whether he or she needs additional training in a certain area. While it is important for the laboratory supervisor to reward advancement, it is also important that the supervisor identify those for whom progress has become static or those who need additional mentorship. ${ }^{3}$ The laboratory supervisor is responsible for thoroughly evaluating and approving medical laboratory scientists' performance so that they can proceed to the next competency level. Al- 
Table 2. Requirements for Discovery Level

Displays Operational Skills on Automated Hematology Analyzers in the Inner Loop, Including Quality Control, Patient Testing, Data Interpretation, and Routine Maintenance

- Run quality control and review two weeks of quality control data for appropriate documentation and corrective action

- Perform analysis of patient blood samples for 10 days and is able to discuss and resolve problematic specimens, ensuring valid results

- Checks pending samples throughout and at the end of the shift

- Examines 10 laboratory information system (LIS) or automated hematology analyzer printouts with discrepant results

- Performs daily and weekly maintenance of the analyzers and checks their function

- Performs basic troubleshooting, such as calling the service hotline, as needed

Displays Operational Skills on All Chemistry Analyzers, Including Quality Control, Patient Testing, Data Interpretation, and Routine Maintenance

- Run quality control and review 2 weeks of quality control data on each chemistry analyzer for appropriate documentation and corrective action

- Performs analysis of patient specimens (including urine, cerebrospinal fluid [CSF], and body fluids) for 10 days and is able to discuss and resolve problematic specimens, ensuring valid results

- Checks pending samples throughout and at the end of the shift

- Examines 10 LIS or chemistry analyzer printouts with discrepant results from each analyzer

- Perform daily and weekly maintenance and analyzer function checks on each analyzer

- Perform basic troubleshooting including calling the service hotline as needed

Displays Operational Skills on Automated Coagulation Analyzers and Thromboelastography, Including Quality Control, Patient Testing, Data Interpretation, and Routine Maintenance

- Runs quality control and review 2 weeks of quality control data for appropriate documentation and corrective action

- Perform analysis of patient samples for 10 days and is be able to discuss and resolve problematic specimens, ensuring valid results

- Check pending samples throughout and at the end of the shift

- Examines 10 LIS or coagulation analyzer printouts with discrepant results

- Performs daily and weekly maintenance analyzer and checks their function

- Performs basic troubleshooting including calling the service hotline, as needed

Displays Operational Skills on Automated and Manual Urine Analyzers, Osmometer, and Fecal Occult Blood, including Quality Control, Patient Testing, Data Interpretation, and Routine Maintenance

- Runs quality control and reviews 2 weeks of quality control data for appropriate documentation and corrective action

- Performs analysis of patient specimens for 5 days and is able to discuss and resolve problematic specimens, ensuring valid results

- Displays operational skill in performing of urine human chorionic gonadotropin (hCG) tests and preparing of body fluid crystal slides

- Checks pending samples throughout and at the end of the shift

- Examines 10 LIS or urinalysis analyzer printouts with discrepant results

- Performs daily and weekly maintenance of the analyzers and checks their function

- Performs basic troubleshooting such as calling the service hotline, as needed

Perform Manual Blood Differentials Counts

- Performs manual blood differential count on at least 25 normal and 25 abnormal slides

- Examines at least 10 peripheral smears, identifying cellular and noncellular components and describing how they relate to disease states

Efficiently Utilizes LIS and Electronic Medical Record System to Report and Document Patient Data, Communications, Quality Control, and Error Correction

- Accurate, complete reporting and documentation is observed by the laboratory supervisor in 1 week's worth of data generation

- Passes an examination consisting of 20 questions about the LIS

Processes Specimens from Other Laboratories, Clinics, and Patient Floors into the LIS

- Processes at least 50 specimens into the LIS and monitors them for accuracy and functionality

- Observes the processing testing of reference lab specimens and the results of these tests

Appropriately Notifies Practitioner of Critical Values, Including Meeting Read-Back, Timing, and Documentation

- Notifies the practitioner

- Notifies the practitioner in a timely manner

- Appropriately documents notification in LIS

Obtain 6.0 Hours of Continuing Education Credit Per Year

- Participates in audio/video conferences, seminars, workshops, or faculty lectures

- Accurately documents completion of continuing education hours 


\section{CLINICAL PRACTICE}

Table 3. Requirements for Application Level

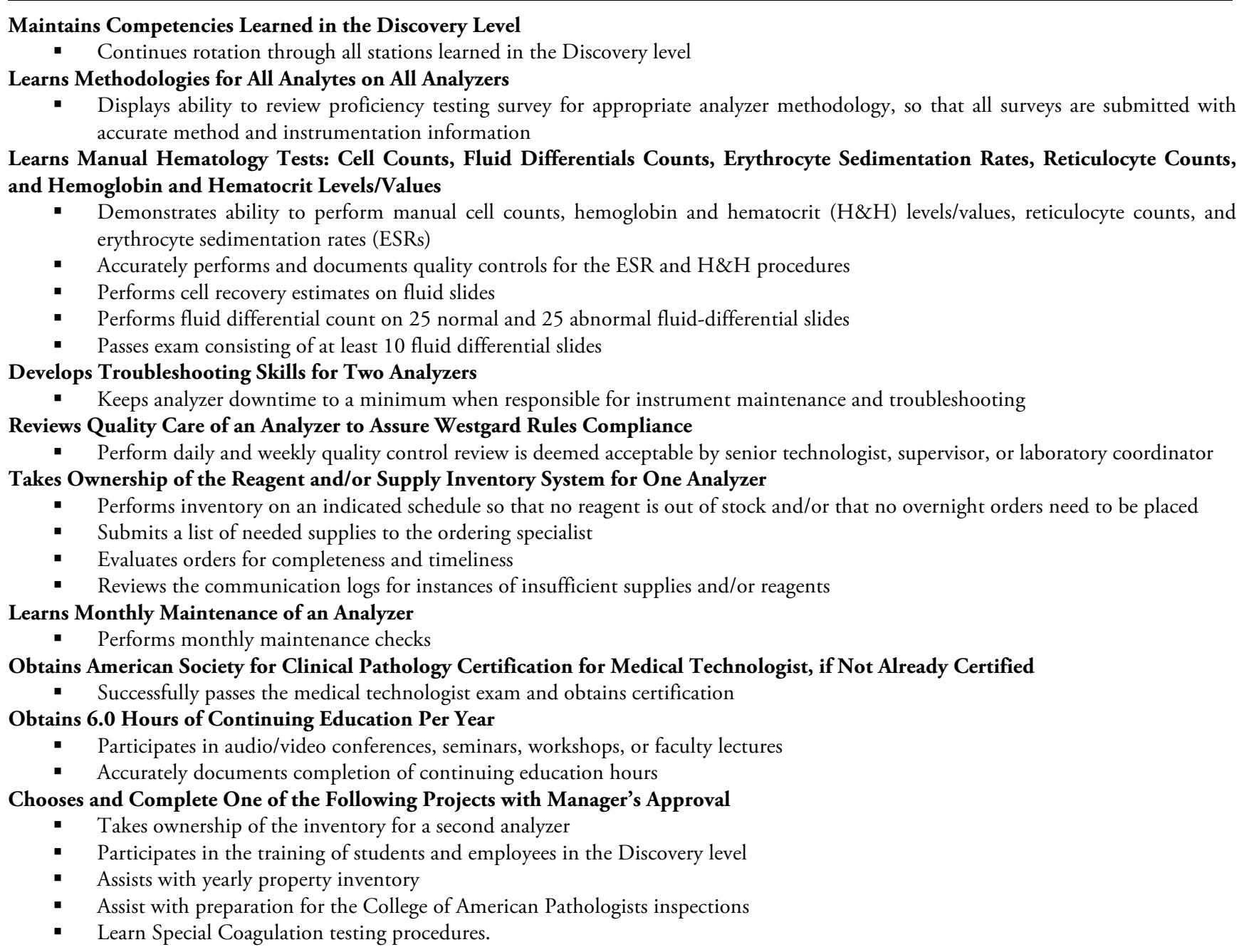

though a supervisor oversees the PDM, it is the responsibility of the medical laboratory scientist to take the necessary steps to complete all the requirements for his or her current competency level.

\section{Compensation}

In order to be eligible for additional monetary compensation (over and above the adjustments made for cost of living and/or years of service), medical laboratory scientists must complete all the requirements of their competency levels within the designated time frame. If additional time is needed to complete a level because of extenuating circumstances beyond the control of the technologist, approval must be obtained from a laboratory manager or departmental administrator. Monetary compensation includes an increase in base salary, a one-time payout, or a combination of both, depending on the competency level completed and the employee's current base salary. No adjustment is made to the base salary if the medical technologist's current base salary exceeds the maximum for that competency level. Compensation adjustments are made biannually.

\section{CONCLUSIONS}

By implementing the PDM, the MD Anderson Core Laboratory has set forth a framework for development of laboratory professionalism that exceeds performing routine laboratory duties. The PDM creates an opportunity for medical laboratory scientists to expand their skills and abilities in a laboratory, as well as acquire an overall understanding of the requirements for making the laboratory functional. The PDM encourages continuous learning of new tasks and skill 
Table 4. Requirements for Maturation Level

\section{Continues to Build on Skills Learned in the Discovery and Application Levels}

- Applies current policies and procedures to all aspects of work with less than or equal to two Sentinel Event errors per year. These errors require an Occurrence Report and are classified as potentially harmful to the patient

Reviews and Revises Current Procedures; Writes New Procedures

- Revisions and the new procedures must meet approval by supervisor, coordinator, manager, and medical director

Assists with Preparation for the College of American Pathologists Inspections

- Successfully passes the online College of American Pathologists (CAP) inspector training course

- Participates in the mock-CAP inspection of the Core Laboratory and other laboratories

- Assists with preparation for the CAP inspection in the main laboratories and other laboratories

- Takes an active part in CAP inspection, answering questions as needed

- Attends CAP inspections (with Manager's approval)

- Assists with our CAP proficiency testing

Obtains 8.0 Hours of Continuing Education Per Year

- Participates in audio/video conferences, seminars, workshops, or faculty lectures

- Accurately documents completion of continuing education hours

Perform Semiannual Linearity and Correlation Checks on Designated Analyzer

- Performs linearity and correlation checks in an accurate and timely manner

- Accurately analyzes and interprets data

- Investigates and resolves any problems detected

Chooses and Completes Two of the Following Projects with Manager's Approval

- Assists with laboratory information system (LIS) testing of current and new procedures

- Develops troubleshooting skills for two additional analyzers

- Becomes qualified as a differential reviewer

- Assists the Safety Officer

- Assists with Quality Assurance/Performance Improvement (QA/PI) projects

- Reviews quality care of an analyzer to assure compliance Westgard rules

- Assists with calibration of an analyzer

Table 5. Requirements for Expert Level

\section{Continues to Build on Skills Learned in the Discovery, Application, and Maturation Levels}

- Applies current policies and procedures to all aspects of work with less than or equal to two Sentinel Event errors per year. These errors require an Occurrence Report and are classified as potentially harmful to the patient

\section{Demonstrates Ability to Assume Supervisor's Duties in His or Her Absence}

- Operates the laboratory in a professional manner while responsible for the laboratory

- Receives zero or no complaints from administration or customers while responsible for the laboratory

Conducts Root Cause Analysis as part of Quality Assurance/Performance Improvement investigation

- Study and report outcome of root cause analysis investigation to administration for acceptability

Obtains 8.0 Hours of Continuing Education Per Year

- Participates in audio/video conferences, seminars, workshops, or faculty lectures

- Accurately documents completion of continuing education hours

\section{Assists with Preparation for the College of American Pathologists Inspections}

- Successfully passes the online College of American Pathologists (CAP) inspector training course

- Participates in mock-CAP inspection of the Core Laboratory and other laboratories

- Assist with preparation for CAP inspection in main laboratories and other laboratories

- Takes an active part in our CAP inspection, answering questions as needed

- Attends CAP inspections (with manager's approval)

- Assists with our CAP proficiency testing

\section{Complete Soft Skill Requirements}

- Teamwork

o Chairs at least one team or group and demonstrates the ability to initiate, develop, manage, and conclude - exhibiting the ability to build relationships and network

- Conflict resolution

o Demonstrates the ability to resolve personnel conflict issues independently, following the appropriate chain of command when necessary 


\section{CLINICAL PRACTICE}

- Initiative

o Demonstrate the ability to take action without being prompted on issues such as quality control (QC), maintenance, quality assurance (QA), scheduling, workflow, staffing, and patient care issues

- Analytical thinking

o Oversees at least one Lean/root-cause-analysis initiative, demonstrating analytical thinking while gathering relevant information, breaking down and mapping current work flow, and following up on completion of a successful project

- Communication

o Demonstrates the ability to independently request or state information using appropriate grammar, format, and context for the audience. Prepares reports or proposals using the appropriate format

- Coach and develop

o Demonstrates the ability to mentor employees, coach appropriately, and model professionalism. Initiates addressing employee performance problems, drafting improvement plans, coaching staff, and monitoring results

- Publications and presentations

o Participates in laboratory meetings, in-service meetings, attends workshops, and gives presentations or presents publications at a national or local meeting

Consults with Laboratory Manager and Chooses One of the Following Tracks

- Technical track: expertise in chemistry or hematology

o Attends analyzer school or off-site workshops or user classes

o Works with service engineers to troubleshoot problems

o Consults with service hotline as needed

o Become a differential reviewer if hematology is chosen

o Instructs staff on correct maintenance and troubleshooting techniques

o Work on a new instrument evaluation

- Management track

o Takes the six management classes offered

o Continues the CAP inspection process outlined in the Maturation level

o Continues working with the QA/PI projects as outlined in the Maturation level; Develops new QA/PI projects as needed, and attends the monthly QA/PI meeting

o Develops or revises competency tests as needed

o Option: acquires authorization privilege for either timekeeping or purchasing system, depending on the needs of the laboratory and with the approval of the manager

- Education track

o Maintains expertise in all areas to be able to teach students and new employees

o Works with scheduling supervisor to create training schedules

o Develops or revises and presents a lecture for the clinical laboratory science program

o Develops and gives an in-service training class for the Core Laboratory employees

o Option: prepares for and takes American Society Clinical Pathology specialty exam

- Computer track - database coordinator

o Conducts yearly laboratory information system (LIS) QA projects as mandated by CAP

o Performs LIS testing of revised procedures

o Performs LIS testing of new procedures

o Maintains accurate LIS database coordination records for the CAP inspection

sets that help medical laboratory scientists become more capable employees. The PDM helps medical laboratory scientists attain an in-depth knowledge of the managerial aspects of a laboratory which helps them work more efficiently. Lastly, the PDM acknowledges the achievements of the medical laboratory scientists, helps them feel more confident in their work.

This article was supported in part by the National Institutes of Health through MD Anderson's Cancer Center Support Grant CA016672

\section{REFERENCES}

1. Lennon PA, Lovshe D, Deleon E, Reynolds A, Pulido L, Cheong S, et al. Professional development model for cytogenetic technologists at The University of Texas MD Anderson Cancer Center sets high standards. J Assoc Genet Technol. 2010;36:195-7.

2. Lennon PA, Deleon E, Reynolds A, Pulido L, Lewing C, Mehta $\mathrm{P}$, et al. A model for rewarding professional growth in the diagnostic molecular oncology laboratory. J Assoc Genet Technol. 2011;37:80-3.

3. Johnson J, Billingsley M, Crichlow T, Ferrell E. Professional development for nurses: mentoring along the U-shaped curve. Nurs Adm Q. 2011;35:119-25. 